\title{
The Covariant Perturbative Approach to Cosmic Microwave Background Anisotropies
}

\author{
Anthony Challinor* \\ Astrophysics Group, Cavendish Laboratory, Madingley Road, \\ Cambridge CB3 OHE, UK.
}

\begin{abstract}
The Ehlers-Ellis $1+3$ formulation of covariant hydrodynamics, when supplemented with covariant radiative transport theory, gives an exact, physically transparent description of the physics of the cosmic microwave background radiation (CMB). Linearisation around a Friedmann-Robertson-Walker (FRW) universe provides a very direct and seamless route through to the linear, gaugeinvariant perturbation equations for scalar, vector and tensor modes in an almost-FRW model. In this contribution we review covariant radiative transport theory and its application to the perturbative method for calculating and understanding the anisotropy of the CMB. Particular emphasis is placed on the inclusion of polarization in a fully covariant manner. With this inclusion, the covariant perturbative approach offers a complete description of linearised CMB physics in an almost-FRW universe.
\end{abstract}

\section{Introduction}

Given the potential impact of observations of the cosmic microwave background radiation on our understanding of the large scale properties of the universe, there is a strong case for developing a physically transparent and portable formalism for the study of CMB physics. Ideally, such a framework should allow one to give: (i) an exact, but physically illuminating description of all the relevant physics; (ii) a simple, gauge-invariant linear perturbation theory around a variety of background models. Along with many other workers in this field [1]-[7], we have found the $1+3$ covariant formulation of hydrodynamics [8, 9] and radiative transport theory [10, 11] to be well suited to this task.

*A.D.Challinor@mrao.cam.ac.uk 
In this contribution we begin by reviewing the exact $1+3$ covariant theory of polarized radiative transport in the presence of Thomson scattering (the dominant scattering mechanism for CMB photons over the epoch of interest). This work builds on the covariant approach to kinetic theory developed in [10, 11]. The angular dependence of the intensity and polarization are conveniently described in terms of projected symmetric trace free (PSTF) tensors. The multipole equations governing the dynamics of these radiation variables are given in linearised form around an almost-FRW universe, and generic features of their solution are discussed qualitatively. By further decomposing the linearised multipole equations in harmonic functions, we arrive at a complete set of gauge-invariant perturbation equations in a form which is convenient for numerical implementation, but which still preserves the physical transparency of the $1+3$ covariant approach. We present the modeexpanded perturbation equations for scalar (density clumping) modes, and discuss briefly their numerical implementation and some numerical results.

We employ standard general relativity and adopt a $(+---)$ signature for the metric $g_{a b}$. Our conventions for the Riemann and Ricci tensors are fixed by $\left[\nabla_{a}, \nabla_{b}\right] u_{c}=R_{a b c}{ }^{d} u_{d}$ and $R_{a c}=R_{a b c}{ }^{b}$. The spacetime alternating tensor is denoted $\eta_{a b c d}$. Round brackets denote symmetrisation on the enclosed indices, square brackets denote antisymmetrisation, and angle brackets denote the PSTF part (the projection is with $h_{a b}=g_{a b}-u_{a} u_{b}$, where $u_{a}$ is the fundamental velocity of the $1+3$ approach). Overdots denote the action of $u^{a} \nabla_{a}$, and the totally projected covariant "derivative" is denoted by $D_{a}$.

\section{Covariant Radiative Transport Theory}

In the $1+3$ covariant approach to cosmology (see [12 for a recent review), the cosmological model is described in terms of geometric variables that have a direct interpretation in terms of the observations of a preferred set of observers who are comoving with the fundamental velocity field $u^{a}$. We shall refer to the choice of $u^{a}$ as a frame choice円. We consider a diffuse radiation field (the CMB) observed from the $u^{a}$ frame. The Stokes parameters of the radiation in some solid angle $d \Omega$ and some photon energy range $d E$, along the unit projected vector $e^{a}$, can be combined into a second-rank polarization tensor $P_{a b}(E, e)$ which is projected relative to $u^{a}$ and $e^{a}$ on both indices. The non-vanishing components of $P_{a b}(E, e)$ on an orthonormal tetrad with the 0 -direction aligned with $u^{a}$ and the 3 -direction with $e^{a}$ are defined

\footnotetext{
${ }^{1}$ The issue of how to specify $u^{a}$ in an appropriate physical manner is discussed in $[7,12]$.
} 
in terms of the Stokes parameters via:

$$
\begin{aligned}
& P_{11}=\frac{1}{2}[I(E, e)+Q(E, e)] \\
& P_{12}=\frac{1}{2}[U(E, e)+V(E, e)] \\
& P_{21}=\frac{1}{2}[U(E, e)-V(E, e)] \\
& P_{22}=\frac{1}{2}[I(E, e)-Q(E, e)] .
\end{aligned}
$$

Our notation for the Stokes parameters follows [13]. The transformation properties of the Stokes parameters under rotation of the 1- and 2-directions ensures that $P_{a b}(E, e)$, as defined, is tensor-valued. Introducing a screen projection tensor $\mathcal{H}_{a b}$,

$$
\mathcal{H}_{a b} \equiv h_{a b}+e_{a} e_{b}
$$

the polarization tensor can be written in irreducible form

$$
P_{a b}(E, e)=-\frac{1}{2} I(E, e) \mathcal{H}_{a b}+\mathcal{P}_{a b}(E, e)-\frac{1}{2} V(E, e) \epsilon_{a b c} e^{c},
$$

where $\epsilon_{a b c} \equiv \eta_{a b c d} u^{d}$ and $\mathcal{P}_{a b}(E, e)$ is a PSTF tensor which is orthogonal to $e^{a}$ :

$$
\mathcal{P}_{a b}(E, e)=\mathcal{H}_{a}^{c} \mathcal{P}_{c b}(E, e)
$$

\subsection{The Multipole Decomposition}

For many applications it is convenient to describe the angular dependence of the polarization tensor in terms of PSTF tensor-valued multipole moments. Since $I(E, e)$ and $V(E, e)$ are scalar fields on the sphere they can be expanded as follows:

$$
\begin{gathered}
I(E, e)=\sum_{l=0}^{\infty} I_{A_{l}}(E) e^{A_{l}} \\
V(E, e)=\sum_{l=0}^{\infty} V_{A_{l}}(E) e^{A_{l}},
\end{gathered}
$$

where $I_{A_{l}}(E)$ and $V_{A_{l}}(E)$ are energy-dependent PSTF tensors, and we have introduced the index notation $A_{l} \equiv a_{1} \ldots a_{l}$ with $e^{A_{l}} \equiv e^{a_{1}} \ldots e^{a_{l}}$. The expansions of $I(E, e)$ and $V(E, e)$ can be inverted to determine the multipoles, for example

$$
I_{A_{l}}(E)=\frac{1}{\Delta_{l}} \int d \Omega I(E, e) e_{\left\langle A_{l}\right\rangle}, \quad \text { with } \quad \Delta_{l} \equiv \frac{4 \pi(-2)^{l}(l !)^{2}}{(2 l+1) !} .
$$

For the linear polarization tensor $\mathcal{P}_{a b}(E, e)$ we must expand in tensor spherical harmonics that are projected perpendicular to $e^{a}$ (tensors projected perpendicular 
to $e^{a}$, such as $\mathcal{P}_{a b}(E, e)$, are denoted transverse in [14]). It is most convenient to use pure-spin PSTF harmonics [14], so that

$$
\mathcal{P}_{a_{1} a_{2}}(E, e)=\sum_{l=2}^{\infty}\left[\mathcal{E}_{a_{1} a_{2} B_{l-2}} e^{B_{l-2}}\right]^{T T}+\sum_{l=2}^{\infty}\left[e_{c_{1}} \epsilon^{c_{1} c_{2}}{ }_{\left(a_{1}\right.} \mathcal{B}_{\left.a_{2}\right) c_{2} B_{l-2}} e^{B_{l-2}}\right]^{T T} .
$$

Coordinate-dependent versions of this expansion were first discussed in the context of all-sky CMB polarization data in [15, 16]. In equation (8) we have left the energy dependence of the PSTF tensors $\mathcal{E}_{A_{l}}(E)$ and $\mathcal{B}_{A_{l}}(E)$ implicit. The superscript $T T$ denotes the transverse traceless part:

$$
\left[A_{a b}\right]^{T T} \equiv \mathcal{H}_{a}^{c_{1}} \mathcal{H}_{b}^{c_{2}} A_{c_{1} c_{2}}-\frac{1}{2} \mathcal{H}_{a b} \mathcal{H}^{c_{1} c_{2}} A_{c_{1} c_{2}}
$$

where $A_{a b}$ is a second-rank tensor. The two summations in equation (8) define the electric and magnetic parts of $\mathcal{P}_{a b}(E, e)$. The $l$-th term in the electric part has parity $(-1)^{l}$, while the $l$-th term in the magnetic part has parity $(-1)^{l-1}$. The electric and magnetic multipoles can be determined from the polarization tensor with the following inversion formulae:

$$
\begin{aligned}
\mathcal{E}_{A_{l}}(E) & =\frac{1}{\Delta_{l}} \frac{2 l(l-1)}{(l+1)(l+2)} \int d \Omega e_{\left\langle A_{l-2}\right.} \mathcal{P}_{\left.a_{l} a_{l-1}\right\rangle}(E, e), \\
-\mathcal{B}_{A_{l}}(E) & =\frac{1}{\Delta_{l}} \frac{2 l(l-1)}{(l+1)(l+2)} \int d \Omega e_{b} \epsilon^{b c}{ }_{\left\langle a_{l}\right.} e_{A_{l-2}} \mathcal{P}_{\left.a_{l-1}\right\rangle c}(E, e),
\end{aligned}
$$

which follow from the orthogonality of the $e_{\left\langle A_{l}\right\rangle}$. We shall also make use of the energy-integrated multipoles, $I_{A_{l}}, \mathcal{E}_{A_{l}}, \mathcal{B}_{A_{l}}$ and $V_{A_{l}}$, where, for example,

$$
I_{A_{l}} \equiv \Delta_{l} \int d E I_{A_{l}}(E)
$$

It follows that the three lowest energy-integrated multipoles of $I(E, e)$ give the radiation energy density, momentum density and anisotropic stress respectively:

$$
I=\rho^{(\gamma)}, \quad I_{a}=q_{a}^{(\gamma)}, \quad I_{a b}=\pi_{a b}^{(\gamma)} .
$$

The scale dependence of intensity and polarization correlations on the CMB sky are described by their angular power spectra. In a statistically isotropic ensemble of CMB skies, the power spectrum of intensity anisotropies $C_{l}^{I I}$ is defined by the ensemble average [6]

$$
\left(\frac{\pi}{I}\right)^{2}\left\langle I_{A_{l}} I^{B_{l^{\prime}}}\right\rangle=\Delta_{l} C_{l}^{I I} \delta_{l l^{\prime}} h_{\left\langle A_{l}\right\rangle}^{\left\langle B_{l}\right\rangle}
$$

where $h_{\left\langle A_{l}\right\rangle}^{\left\langle B_{l}\right\rangle} \equiv h_{\left\langle a_{1}\right.}^{\left\langle b_{1}\right.} \ldots h_{\left.a_{l}\right\rangle}^{\left.b_{l}\right\rangle}$. In the limit of almost-isotropic radiation, the $I_{A_{l}}$ are related to the gauge-invariant bolometric temperature perturbation (from the allsky mean) $\delta_{T}(e)$ via [2]

$$
\delta_{T}(e)=\frac{\pi}{I} \sum_{l=1}^{\infty} \frac{1}{\Delta_{l}} I_{A_{l}} e^{A_{l}}
$$


from which we recover the usual Legendre expansion of the temperature correlation function:

$$
\left\langle\delta_{T}(e) \delta_{T}\left(e^{\prime}\right)\right\rangle=\sum_{l=1}^{\infty} \frac{(2 l+1)}{4 \pi} C_{l}^{I I} P_{l}\left(-e^{a} e_{a}^{\prime}\right) .
$$

Power spectra for the polarization and the cross-correlation between electric polarization and intensity anisotropies can be defined in a similar manner to $C_{l}^{I I}$. However, for consistency with previous approaches based on an expansion in spinweighted harmonics [16], with the normalisation conventions adopted here it is necessary to include an additional factor of $\sqrt{ }[l(l-1) /(l+1)(l+2)]$ in the definition of $C_{l}$ for each factor of the polarization. For example,

$$
\left(\frac{\pi}{I}\right)^{2}\left\langle\mathcal{E}_{A_{l}} \mathcal{E}^{B_{l^{\prime}}}\right\rangle=\frac{l(l-1)}{(l+2)(l+1)} \Delta_{l} C_{l}^{\mathcal{E} \mathcal{E}} \delta_{l l^{\prime}} h_{\left\langle A_{l}\right\rangle}^{\left\langle B_{l}\right\rangle} .
$$

Note there cannot be any correlation between $\mathcal{B}_{A_{l}}$ and either of $\mathcal{E}_{A_{l}}$ or $I_{A_{l}}$ in a parity symmetric ensemble. As noted in [16], the definitions of the polarization power spectra adopted here are independent of the choice of polarization basis vectors, which has significant advantages over correlating the Stokes parameters directly.

\subsection{The Boltzmann Equation}

The evolution of the polarization tensor $P_{a b}(E, e)$ along the path of the radiation in phase space is described by the (collisional) Boltzmann equation

$$
\mathcal{L}\left[E^{-3} P_{a b}(E, e)\right]=K_{a b}(E, e) .
$$

The appropriate Liouville operator $\mathcal{L}$ for the transverse tensor $P_{a b}(E, e)$ acts according to

$$
\mathcal{L} P_{a b}(E, e)=\mathcal{H}_{a}^{c_{1}} \mathcal{H}_{b}^{c_{2}} p^{d} \nabla_{d} P_{c_{1} c_{2}}(E, e)
$$

where the derivative is along the photon path $\left\{x^{a}(\lambda), p^{a}(\lambda)\right\}$ in phase space. Here, $p^{a}=E\left(u^{a}+e^{a}\right)$ is the photon momentum and $\lambda$ is an affine parameter with $p^{a}=$ $d x^{a} / d \lambda$. The scattering tensor $K_{a b}(E, e)$ on the right-hand side of equation (18) is transverse. Its exact form for Thomson scattering is given in Section 2.2.2.

The irreducible decomposition of $P_{a b}(E, e)$ into $I(E, e), V(E, e)$ and $\mathcal{P}_{a b}(E, e)$, given as equation (3), is preserved by the action of the Liouville operator, so that

$$
\begin{aligned}
\mathcal{L}\left[E^{-3} P_{a b}(E, e)\right]=-\frac{1}{2} \frac{d}{d \lambda}\left[E^{-3} I(E, e)\right] \mathcal{H}_{a b} & +\mathcal{L}\left[E^{-3} \mathcal{P}_{a b}(E, e)\right] \\
& -\frac{1}{2} \frac{d}{d \lambda}\left[E^{-3} V(E, e)\right] \epsilon_{a b c} e^{c} .
\end{aligned}
$$

In the absence of scattering $\left(K_{a b}(E, e)=0\right)$, we recover the well-known results that the occupation number $I(E, e) / E^{3}$, degree of circular polarization $V(E, e) / I(E, e)$, and degree of linear polarization $\sqrt{ }\left[2 \mathcal{P}_{a b}(E, e) \mathcal{P}^{a b}(E, e)\right] / I(E, e)$ are independently conserved along the path of the radiation. 


\subsubsection{Transformation Laws Under Changes of Frame}

The $1+3$ covariant approach considers observations made from the viewpoint of a set of observers moving with the fundamental velocity $u^{a}$. Often, it is useful to have available the transformation laws relating these observations to those made with respect to a different velocity field $\tilde{u}^{a}=\gamma\left(u^{a}+v^{a}\right)$. Here, $v^{a}$ is the projected relative velocity (in the $u^{a}$ frame), and $\gamma$ is the associated Lorentz factor.

For a given photon momentum $p^{a}=E\left(u^{a}+e^{a}\right)$, the energy $\tilde{E}$ and direction of propagation $\tilde{e}^{a}$ in the $\tilde{u}^{a}$ frame are given by

$$
\begin{aligned}
\tilde{E} & =\gamma E\left(1+e^{a} v_{a}\right) \\
\tilde{e}^{a} & =\left[\gamma\left(1+e^{b} v_{b}\right)\right]^{-1}\left(e^{a}+u^{a}\right)-\gamma\left(u^{a}+v^{a}\right) .
\end{aligned}
$$

The screen projection tensor $\tilde{\mathcal{H}}_{a b}$ in the $\tilde{u}^{a}$ frame is related to the equivalent tensor in the $u^{a}$ frame by

$$
\tilde{\mathcal{H}}_{a b}=\mathcal{H}_{a b}-\frac{2 \gamma}{\tilde{E}} p_{(a} \mathcal{H}_{b) c} v^{c}+\left(\frac{\gamma}{\tilde{E}}\right)^{2} p_{a} p_{b} \mathcal{H}^{c_{1} c_{2}} v_{c_{1}} v_{c_{2}} .
$$

The polarization tensor in the $\tilde{u}^{a}$ frame, $\tilde{P}_{a b}(\tilde{E}, \tilde{e})$, is related to $P_{a b}(E, e)$ by

$$
\tilde{E}^{-3} \tilde{P}_{a b}(\tilde{E}, \tilde{e})=E^{-3} \tilde{\mathcal{H}}_{a}^{c_{1}} \tilde{\mathcal{H}}_{b}^{c_{2}} P_{c_{1} c_{2}}(E, e) .
$$

From this transformation law we recover the frame-invariance of $I(E, e) / E^{3}$, and the degrees of circular and linear polarization.

It is straightforward to verify that under changes of frame the action of the Liouville operator transforms according to

$$
\tilde{\mathcal{L}}\left[\tilde{E}^{-3} \tilde{P}_{a b}(\tilde{E}, \tilde{e})\right]=\tilde{\mathcal{H}}_{a}^{c_{1}} \tilde{\mathcal{H}}_{b}^{c_{2}} \mathcal{L}\left[E^{-3} P_{c_{1} c_{2}}(E, e)\right],
$$

so that the collision tensor in the Boltzmann equation must transform as

$$
\tilde{K}_{a b}(\tilde{E}, \tilde{e})=\tilde{\mathcal{H}}_{a}^{c_{1}} \tilde{\mathcal{H}}_{b}^{c_{2}} K_{c_{1} c_{2}}(E, e) .
$$

This transformation law provides a useful means of generating the scattering tensor for a general choice of frame given its form in some particular frame. (It is often the case that the scattering process picks out a frame in which $K_{a b}(E, e)$ is particularly simple.) The transformation laws for the radiation multipoles $I_{A_{l}}(E), V_{A_{l}}(E), \mathcal{E}_{A_{l}}(E)$ and $\mathcal{B}_{A_{l}}(E)$ are non-local in energy and show multipole coupling due to relativistic beaming. Since the detailed form of the multipole transformation laws is not needed for the calculation of linearised CMB anisotropies and polarization, we shall not give them here. 


\subsection{2 $\quad K_{a b}(E, e)$ for Thomson Scattering}

Over the epoch of interest for the generation of CMB anisotropies and polarization, the dominant scattering mechanism for CMB photons is Compton scattering off free electrons. Well after electron/positron annihilation it is a good approximation to ignore electron recoil and the small thermal effects due to the electron distribution function. In this limit it is convenient to work initially in the rest frame of the tightly-coupled electron/baryon plasma, the four-velocity of which we denote by $\tilde{u}^{a}$.

The scattering tensor $K_{a b}(E, e)$ can be deduced from the standard result for the Thomson scattering kernel expressed in terms of the Stokes parameters (see, for example, [17]). Assuming an unpolarized distribution of electrons, we find the following expression for the scattering tensor in the $\tilde{u}^{a}$ frame in $1+3$ covariant irreducible form:

$$
\begin{aligned}
\tilde{E}^{2} \tilde{K}_{a b}(\tilde{E}, \tilde{e})= & n_{e} \sigma_{\mathrm{T}}\left\{-\frac{1}{2} \tilde{\mathcal{H}}_{a b}[-\tilde{I}(\tilde{E}, \tilde{e})+\tilde{I}(\tilde{E})\right. \\
& \left.+\frac{1}{10} \tilde{I}_{c_{1} c_{2}}(\tilde{E}) \tilde{e}^{c_{1}} \tilde{e}^{c_{2}}+\frac{3}{5} \tilde{\mathcal{E}}_{c_{1} c_{2}}(\tilde{E}) \tilde{e}^{c_{1}} \tilde{e}^{c_{2}}\right] \\
& +\left[-\tilde{\mathcal{P}}_{a b}(\tilde{E}, \tilde{e})+\frac{1}{10}\left[\tilde{I}_{a b}(\tilde{E})\right]^{T T}+\frac{3}{5}\left[\tilde{\mathcal{E}}_{a b}(\tilde{E})\right]^{T T}\right] \\
& \left.-\frac{1}{2} \tilde{\epsilon}_{a b c} \tilde{e}^{c}\left[-\tilde{V}(\tilde{E}, \tilde{e})+\frac{1}{2} \tilde{V}_{d}(\tilde{E}) \tilde{e}^{d}\right]\right\},
\end{aligned}
$$

where $n_{e}$ is the electron number density in the $\tilde{u}^{a}$ frame, and $\sigma_{T}$ is the Thomson cross section. Equation (27) is an exact expression for the scattering tensor in the rest frame of the electrons. The first set of terms in square brackets source the intensity evolution, the second set source the evolution of the linear polarization, while the final set source the circular polarization. Note that the source terms for the intensity have no monopole $(l=0)$ moment in the $\tilde{u}^{a}$ frame, since there is no energy transfer in the Thomson limit in the electron rest frame. Note also how the quadrupole $(l=2)$ moment of the electric polarization sources the intensity, and the quadrupole moment of the intensity sources the electric polarization. (It is for this reason that polarization should be included in accurate CMB codes even if only the temperature information is of interest.) Equation (27) is equivalent to the scattering terms derived recently within the context of the "total angular momentum" advocated by $\mathrm{Hu}$ and collaborators [18, 19]. However, unlike the results in [18, 19], equation (27) is free from any (spatial) harmonic decomposition of the radiation variables.

Transforming the scattering term to an arbitrary frame, and integrating over energies, we find the exact evolution of the intensity:

$$
\begin{aligned}
\int d E E^{2} \mathcal{L}\left[E^{-3} I(E, e)\right]= & -n_{e} \sigma_{\mathrm{T}} \gamma\left(1+e^{a} v_{a}\right) \int d E I(E, e) \\
& +\frac{1}{4 \pi} n_{e} \sigma_{\mathrm{T}}\left[\gamma\left(1+e^{a} v_{a}\right)\right]^{-3}\left(\tilde{I}+\tilde{\zeta}_{c_{1} c_{2}} \tilde{e}^{c_{1}} \tilde{e}^{c_{2}}\right),
\end{aligned}
$$


the linear polarization:

$$
\begin{aligned}
\int d E E^{2} \mathcal{L}\left[E^{-3} \mathcal{P}_{a b}(E, e)\right]= & -n_{e} \sigma_{\mathrm{T}} \gamma\left(1+e^{c} v_{c}\right) \int d E \mathcal{P}_{a b}(E, e) \\
& +\frac{1}{4 \pi} n_{e} \sigma_{\mathrm{T}}\left[\gamma\left(1+e^{c} v_{c}\right)\right]^{-3}\left[\tilde{\mathcal{H}}_{a}^{c_{1}} \tilde{\mathcal{H}}_{b}^{c_{2}} \tilde{\zeta}_{c_{1} c_{2}}\right]^{T T}
\end{aligned}
$$

and the circular polarization:

$$
\begin{aligned}
\int d E E^{2} \mathcal{L}\left[E^{-3} V(E, e)\right]= & -n_{e} \sigma_{\mathrm{T}} \gamma\left(1+e^{a} v_{a}\right) \int d E V(E, e) \\
& -\frac{3}{8 \pi} n_{e} \sigma_{\mathrm{T}}\left[\gamma\left(1+e^{a} v_{a}\right)\right]^{-3} \tilde{V}_{b} \tilde{e}^{b} .
\end{aligned}
$$

Here, tildes refer to quantities in the rest frame of the electrons, and

$$
\tilde{\zeta}_{a b} \equiv \frac{3}{4} \tilde{I}_{a b}+\frac{9}{2} \tilde{\mathcal{E}}_{a b}
$$

Expressions for $\tilde{I}, \tilde{I}_{a b}$, and $\tilde{\mathcal{E}}_{a b}$ in terms of quantities in the $u^{a}$ frame can be derived from the multipole transformation laws if required.

Equation (30) demonstrates that circular polarization is not sourced by Thomson scattering off unpolarized electrons, so that in the absence of polarising agents, such as primordial magnetic fields, we should not expect the primordial component of the microwave sky to have any circular polarization. In light of these remarks, we shall not consider the $V$ Stokes parameter any further here. (Some effects of primordial magnetic fields on the CMB are discussed in [20, 21].)

\section{Linearisation Around a FRW Model}

The observed isotropy of the CMB (the anisotropies are at the level of only one part in $10^{5}$ [22]) combines with the Copernican assumption to imply that on large scales the universe is well approximated by perturbing a FRW model [1], 2]. In the $1+3$ covariant and gauge-invariant approach to studying perturbations in cosmology [23, 24, 25], the perturbed model is investigated in terms of covariant variables which are defined in the real universe (as opposed to the background model), and which vanish identically in the background model. With a physical choice for the fundamental velocity $u^{a}$, the approach is gauge-invariant to all orders. Given a smallness parameter $\epsilon$ for the almost-FRW model, the covariant variables that describe inhomogeneity and anisotropy are at most $O(\epsilon)$. Important examples of $O(\epsilon)$ variables in a generic almost-FRW spacetime include the kinematic variables derived 
from $u^{a}$ :

$$
\begin{array}{ll}
\text { shear } & \sigma_{a b}=D_{\langle a} u_{b\rangle} \\
\text { vorticity } & \omega_{a b}=D_{[a} u_{b]} \\
\text { acceleration } & A_{a}=\dot{u}_{a} \\
\text { expansion inhomogeneity } & \mathcal{Z}_{a}=S D_{a} \Theta ;
\end{array}
$$

the matter variables derived from the matter stress-energy tensor $T_{a b}$ :

$$
\begin{array}{lrl}
\text { momentum density } & q_{a}=T_{\langle a\rangle b} u^{b} \\
\text { anisotropic stress } & \pi_{a b}=T_{\langle a b\rangle} \\
\text { density inhomogeneity } & \mathcal{X}_{a}=S D_{a} \rho / \rho ;
\end{array}
$$

the Weyl tensor $C_{a b c d}$ and hence its electric and magnetic parts:

$$
\begin{array}{ll}
\text { electric } & E_{a b}=C_{a c b d} u^{c} u^{d} \\
\text { magnetic } & H_{a b}=-\frac{1}{2} \epsilon_{a c d} C_{b e}{ }^{c d} u^{e} .
\end{array}
$$

Here, $\Theta=\nabla_{a} u^{a}$ is the fractional volume expansion rate, $\rho=T_{a b} u^{a} u^{b}$ is the matter energy density, and $S$ is a covariantly defined scale factor, $\dot{S} / S=\Theta / 3$ with $D_{a} S=O(\epsilon)$. The linearised perturbation equations for the $O(\epsilon)$ variables are derived from the exact $1+3$ covariant hydrodynamic and gravito-electromagnetic equations (see, for example, [12]). The equation set closes when supplemented by constitutive relations for the matter variables.

\subsection{Linearised Multipole Equations}

The equations of radiative transfer provide the constitutive relations for the radiation sector of the cosmological model. It turns out to be most convenient to work directly with the evolution equations for the radiation angular multipoles. The exact multipole equations can be derived from equation (18). The equations for the intensity multipoles $I_{A_{l}}(E)$ are derived in [7, 10, 11]; those for the polarization multipoles are given in [26]. Here, we shall only make use of the linearised multipole equations. Since Thomson scattering does not produce any spectral distortion at linear order (this follows from equation (27)), it is sufficient to consider only the energy-integrated multipoles $I_{A_{l}}, \mathcal{E}_{A_{l}}$ and $\mathcal{B}_{A_{l}}$. Noting that $\mathcal{E}_{A_{l}}$ and $\mathcal{B}_{A_{l}}$ are $O(\epsilon)$ for all $l \geq 2$ in an almost-FRW universe, while $I_{A_{l}}$ are $O(\epsilon)$ for $l>0$, we find the following linearised evolution equations for the electric polarization?:

$$
\begin{aligned}
\dot{\mathcal{E}}_{A_{l}}+\frac{4}{3} \Theta \mathcal{E}_{A_{l}} & +\frac{(l+3)(l-1)}{(l+1)^{2}} D^{b} \mathcal{E}_{b A_{l}}-\frac{l}{(2 l+1)} D_{\left\langle a_{l}\right.} \mathcal{E}_{\left.A_{l-1}\right\rangle} \\
& -\frac{2}{(l+1)} \operatorname{curl} \mathcal{B}_{A_{l}}=-n_{e} \sigma_{\mathrm{T}}\left(\mathcal{E}_{A_{l}}-\frac{2}{15} \delta_{l 2} \zeta_{a b}\right),
\end{aligned}
$$

\footnotetext{
${ }^{2}$ We use the convention that $\mathcal{E}_{A_{l}}$ and $\mathcal{B}_{A_{l}}$ vanish for $l<2$.
} 
and for the magnetic polarization:

$$
\begin{aligned}
\dot{\mathcal{B}}_{A_{l}}+\frac{4}{3} \Theta \mathcal{B}_{A_{l}} & +\frac{(l+3)(l-1)}{(l+1)^{2}} D^{b} \mathcal{B}_{b A_{l}}-\frac{l}{(2 l+1)} D_{\left\langle a_{l}\right.} \mathcal{B}_{\left.A_{l-1}\right\rangle} \\
& +\frac{2}{(l+1)} \operatorname{curl} \mathcal{E}_{A_{l}}=-n_{e} \sigma_{\mathrm{T}} \mathcal{B}_{A_{l}} .
\end{aligned}
$$

Here, the curl of a rank-l tensor $S_{A_{l}}$ is defined by

$$
\operatorname{curl} S_{A_{l}} \equiv \epsilon_{c_{1} c_{2}\left(a_{l}\right.} D^{c_{1}} S_{\left.A_{l-1}\right)}^{c_{2}}
$$

which is PSTF for $S_{A_{l}}$ PSTF. Equations (35) and (36) reveal that $\mathcal{E}_{A_{l}}$ and $\mathcal{B}_{A_{l}}$ are coupled through curl terms (as with Maxwell's equations), and that only the electric quadrupole $\mathcal{E}_{a b}$ has inhomogeneous scattering terms in its evolution equation. These observations are crucial for the prospect of detecting the imprint of primordial gravitational waves on the CMB (see Section 3.2).

The linearised intensity multipole equations are summarised by

$$
\begin{aligned}
\dot{I}_{A_{l}}+\frac{4}{3} \Theta I_{A_{l}}+D^{b} I_{b A_{l}}- & \frac{l}{(2 l+1)} D_{\left\langle a_{l}\right.} I_{\left.A_{l-1}\right\rangle}+\frac{4}{3} I A_{a_{1}} \delta_{l 1}-\frac{8}{15} I \sigma_{a_{1} a_{2}} \delta_{l 2} \\
& =-n_{e} \sigma_{\mathrm{T}}\left(I_{A_{l}}-\frac{4}{3} I v_{a_{1}} \delta_{l 1}-\frac{2}{15} I \zeta_{a_{1} a_{2}} \delta_{l 2}\right), \quad l \geq 1
\end{aligned}
$$

where $v^{a}$ is the projected relative velocity of the electron rest fame. The intensity monopole (radiation energy density) evolves according to

$$
\dot{I}+\frac{4}{3} \Theta I+D^{a} I_{a}=0
$$

which implies the following evolution equation for the comoving projected gradient of the energy density:

$$
\left(S D_{a} I\right)^{\cdot}+\frac{4}{3} \Theta S D_{a} I+S D_{a} D^{b} I_{b}+\frac{4}{3} I \mathcal{Z}_{a}-\frac{4}{3} \Theta S I A_{a}=0 .
$$

The linearised multipole equations (35), (36), (38) and (40) provide a complete description of linearised CMB physics in an almost-FRW universe. From these equations one can infer the model-independent behaviour of the anisotropy and polarization. Prior to recombination, the photon mean free time is short compared to the characteristic expansion time of the universe. On scales much larger than the photon mean free path, the photons are tightly-coupled to the electron/baryon plasma, and the combined system behaves like a fluid. On super-horizon scales $D_{a} I$ grows due to gravitational instability, while on sub-horizon scales power oscillates between $D_{a} I$ and $I_{a}$ (acoustic oscillations). The fluid approximation breaks down inside the diffusion scale [27], where higher-l intensity multipoles are excited at the expense 
of damping the acoustic oscillations. During recombination the mean free path of the photons rises to effectively infinity, with the result that power is transported to higher- $l$ intensity multipoles through the free-streaming projection of $D_{a} I$, the electron peculiar velocity $v_{a}$, and the combination of intensity and electric polarization quadrupoles $\zeta_{a b}$ from the last scattering surface. Power is also transferred to the intensity multipoles through variations of redshift since last scattering along different lines of sight. The electric polarization quadrupole $\mathcal{E}_{a b}$ is generated through recombination from the growing intensity quadrupole anisotropy. This mechanism ceases at last scattering, after which the power in the electric quadrupole is redistributed amongst the higher- $l$ electric and magnetic multipoles via free-streaming. Any reionisation tends to damp the primary intensity anisotropies while boosting the polarization signal, as well as generating new secondary intensity anisotropies (see 28] for a recent review).

\subsection{Scalar Perturbations}

Up to this point our discussion has been concerned with arbitrary (small) perturbations around a FRW model. In particular, unlike most other perturbative approaches (for example, [29]), we have not had to split the perturbations into scalar, vector or tensor modes (see, for example, [30]) to derive the basic, gauge-invariant perturbation equations. However, for detailed calculation it is convenient to make a non-local mode decomposition of the $O(\epsilon)$ covariant variables to decouple the temporal and spatial dependencies of the $1+3$ equations. The decomposition can be performed in linear theory in a fully covariant manner, which leads to a physically transparent, gauge-invariant perturbation theory. Here we shall only give a brief review of the

procedure for scalar modes (those which describe density clumping) [5, 24]. Vector and tensor perturbations are treated in a similar manner.

By definition, in scalar modes the $O(\epsilon)$ variables are constructed from $O(\epsilon)$ scalar potentials. It follows from the constraint equations of $1+3$ covariant hydrodynamics [12 that the vorticity vanishes at $O(\epsilon)$ in a scalar mode. Furthermore, since all curls vanish by construction, the constraint equations of $1+3$ gravitoelectromagnetism [12, 31 constrain the magnetic part of the Weyl tensor to vanish at linear order. The fact that $\operatorname{curl} \mathcal{E}_{A_{l}}$ and $\operatorname{curl} \mathcal{B}_{A_{l}}$ vanish at linear order removes the coupling between the electric and magnetic polarization multipoles in their evolution equations (35) and (36). It follows that the hierarchy for the $\mathcal{B}_{A_{l}}$ has no inhomogeneous terms present, so that scalar modes do not generate magnetic polarization [15, 16. Any detection of primordial magnetic polarization would provide evidence of vector or tensor modes. This proves to be a much more promising approach than relying on their cosmic variance limited temperature signature [32].

The scalar potentials from which the $O(\epsilon)$ covariant variables are derived may be 
expanded in the complete set of scalar eigenfunctions $Q^{(k)}$ of the comoving Laplacian:

$$
D_{a} D^{a} Q^{(k)}=\frac{k^{2}}{S^{2}} Q^{(k)}
$$

with $\dot{Q}^{(k)}=O(\epsilon)$. Rank-l PSTF tensors, which are covariantly constant along the integral curves of $u^{a}$, can be constructed from the $Q^{(k)}$ :

$$
Q_{A_{l}}^{(k)} \equiv\left(\frac{S}{k}\right)^{l} D_{\left\langle a_{1}\right.} \ldots D_{\left.a_{l}\right\rangle} Q^{(k)} .
$$

The $Q_{A_{l}}^{(k)}$ can be used to expand the radiation multipoles, so that, symbolically,

$$
I_{A_{l}}=I \sum_{k} I_{k}^{(l)} Q_{A_{l}}^{(k)}, \quad \mathcal{E}_{A_{l}}=I \sum_{k} \mathcal{E}_{k}^{(l)} Q_{A_{l}}^{(k)}
$$

where the $I_{k}^{(l)}$ and $\mathcal{E}_{k}^{(l)}$ are $O(\epsilon)$ scalars with $O\left(\epsilon^{2}\right)$ projected gradients. It proves convenient to use the notation $I_{k}^{(0)}$ for the scalar coefficients in the expansion of $D_{a} I: \quad D_{a} I=I \sum_{k}(k / S) I_{k}^{(0)} Q_{a}^{(k)}$, so that the mode-expanded intensity multipole equations take the form

$$
\begin{aligned}
\dot{I}_{k}^{(l)}-\frac{k}{S}\left\{\frac{l}{(2 l+1)} I_{k}^{(l-1)}-\frac{(l+1)}{(2 l+1)}\left[1-l(l+2) \frac{K}{k^{2}}\right] I_{k}^{(l+1)}\right\} \\
+\frac{4}{3} \frac{k}{S} A_{k} \delta_{l 1}-\frac{8}{15} \frac{k}{S} \sigma_{k} \delta_{l 2}=-n_{e} \sigma_{\mathrm{T}}\left(I_{k}^{(l)}-\frac{4}{3} v_{k} \delta_{l 1}-\frac{2}{15} \zeta_{k} \delta_{l 2}\right) .
\end{aligned}
$$

Here $A_{k}, \sigma_{k}, v_{k}$ and $\zeta_{k}$ are the dimensionless coefficients in the mode expansions of $A_{a}, \sigma_{a b}, v_{a}$ and $\zeta_{a b}$, and $6 K / S^{2}$ is the zero-order 3 -curvature scalar. The evolution equation for $I_{k}^{(0)}$ follows from equation (40):

$$
\dot{I}_{k}^{(0)}+\frac{k}{S} I_{k}^{(1)}+\frac{4}{3} \frac{k}{S} \mathcal{Z}_{k}-\frac{4}{3} \Theta A_{k}=0
$$

where $\mathcal{Z}_{k}$ are the dimensionless coefficients in the expansion of $\mathcal{Z}_{a}$. For the electric polarization we find

$$
\begin{aligned}
\dot{\mathcal{E}}_{k}^{(l)}-\frac{k}{S}\left\{\frac{l}{(2 l+1)} \mathcal{E}_{k}^{(l-1)}-\frac{(l+3)(l-1)}{(2 l+1)(l+1)}\left[1-l(l+2) \frac{K}{k^{2}}\right] \mathcal{E}_{k}^{(l+1)}\right\} & \\
& =-n_{e} \sigma_{\mathrm{T}}\left(\mathcal{E}_{k}^{(l)}-\frac{2}{15} \zeta_{k} \delta_{l 2}\right) .
\end{aligned}
$$

At this point we have essentially recovered the well-known multipole equations for scalar perturbations in an almost-FRW model [19, 33. Arguably, the equations given here are somewhat more transparent than their gauge-dependent counterparts 


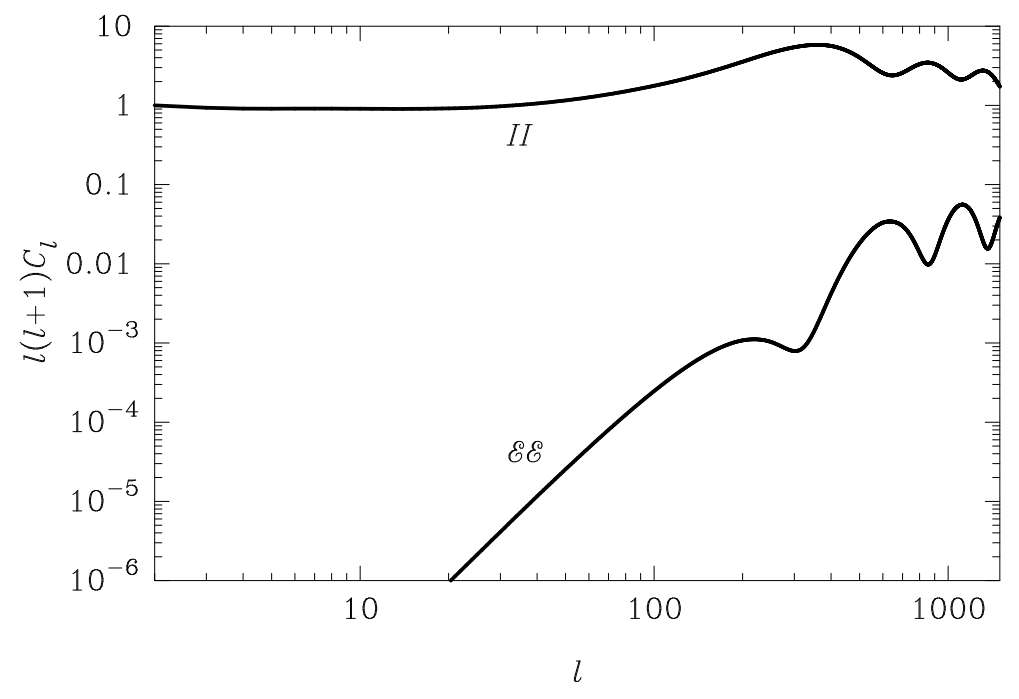

Figure 1: Intensity $C_{l}^{I I}$ and polarization $C_{l}^{\mathcal{E}}$ power spectra from scalar modes in a scale-invariant, adiabatic, open cold dark matter (CDM) model. The baryon fraction is $\Omega_{b}=0.05$, the CDM fraction is $\Omega_{c}=0.4$, the Hubble constant is $H_{0}=50 \mathrm{~km} \mathrm{~s}^{-1} \mathrm{Mpc}^{-1}$, and there is no cosmological constant nor reionisation.

in alternative approaches. For example, the intensity monopole $I_{k}^{(0)}$ describes the spatial inhomogeneity of the radiation energy density, which is of clear physical significance, rather than the gauge-dependent difference between the density in the real universe and the background model. The mode-expanded multipole equations can be integrated directly to determine the anisotropy and polarization for some given initial conditions. To close the equations one must also integrate the modeexpanded hydrodynamical equations for the kinematic variables that source the intensity equations through redshift effects [5]. Since much of the complexity of the coupled multipole equations arises because they describe evolution along the timelike integral curves of $u^{a}$, rather than along the lightlike trajectory of the radiation, it is more efficient to employ integral solutions to the multipole equations rather than integrate a large number of them directly [34]. The integral solutions consist of simple source functions integrated against known geometric projection functions. The integral solutions for scalar and tensor modes are given in [26]; see also [19].

An example of the intensity and polarization power spectra from scalar perturbations in an open model is given in Figure 1. The code to produce the figure was based on CMBFAST [34; the necessary modifications to solve the covariant equations were implemented by Antony Lewis. 


\section{Conclusion}

The $1+3$ covariant formulation of radiative transfer provides a convenient, exact framework within which to study CMB physics. Although we have only considered the linearised form of the transfer equations in our discussion of the anisotropy and polarization in an almost-FRW universe, the formalism is of wider applicability. For example, the approach appears well suited to a systematic study of second-order geometric and scattering effects [7]. In the application to linearised CMB physics in an almost-FRW universe, the $1+3$ covariant perturbative approach shares many of the advantages of the "total angular momentum" method [18, 19]. These include physical transparency, the explicitly quadrupole nature of the radiative source terms in the Boltzmann equation that arise from the angular and polarization dependence of the Thomson cross section, and the direct manner in which the angular multipoles describe the angular scale of anisotropy on the sky. However, the $1+3$ covariant method goes further by ensuring manifest gauge-invariance, by deferring any nonlocal splitting of the perturbations until a late stage of the calculation, and by providing a straightforward linearisation procedure around a variety of background models.

\section{Acknowledgements}

The author would like to thank the organising committee for their invitation to participate in the meeting and Queens' College, Cambridge, for support in the form of a Research Fellowship.

\section{References}

[1] W.R. Stoeger, R. Maartens, and G.F.R. Ellis. Proving almost-homogeneity of the universe: an almost Ehlers-Geren-Sachs theorem. Astrophys. J., 443:1, 1995.

[2] R. Maartens, G.F.R. Ellis, and W.R. Stoeger. Limits on anisotropy and inhomogeneity from the cosmic background radiation. Phys. Rev. D, 51:1525, 1995.

[3] P.K.S. Dunsby. A fully covariant description of CMBR anisotropies. Class. Quantum Grav., 14:3391, 1997.

[4] A.D. Challinor and A.N. Lasenby. Covariant and gauge-invariant analysis of CMB anisotropies from scalar perturbations. Phys. Rev. D, 58:023001, 1998. 
[5] A.D. Challinor and A.N. Lasenby. Cosmic microwave background anisotropies in the CDM model: a covariant and gauge-invariant approach. Preprint astroph/9804301, 1998.

[6] T. Gebbie and G.F.R. Ellis. Covariant cosmic microwave background anisotropies. I: Algebraic relations for mode and multipole representations. Preprint astro-ph/9804316, 1998.

[7] R. Maartens, T. Gebbie, and G.F.R. Ellis. Cosmic microwave background anisotropies: Nonlinear dynamics. Preprint astro-ph/9808163, 1998.

[8] J. Ehlers. Contributions to the relativistic mechanics of continuous media. Gen. Rel. Grav., 25:1225, 1993.

[9] G.F.R. Ellis. Relativistic cosmology. In R.K. Sachs, editor, General Relativity and Cosmology. Proceedings of the International School of Physics, "Enrico Fermi”, Course XLVII, page 104. Academic Press, New York, 1971.

[10] G.F.R. Ellis, D.R. Matravers, and R. Treciokas. Anisotropic solutions of the Einstein-Boltzmann equations: I. General formalism. Ann. Phys., 150:455, 1983.

[11] K.S. Thorne. Relativistic radiative transfer: moment formalisms. Mon. Not. R. Astron. Soc., 194:439, 1981.

[12] G.F.R. Ellis. Cosmological models. Preprint gr-qc/9812040, 1998.

[13] A. Kosowsky. Cosmic microwave background polarization. Ann. Phys., 246:49, 1996.

[14] K.S. Thorne. Multipole expansions of gravitational radiation. Rev. Mod. Phys., 52:299, 1980 .

[15] M. Kamionkowski, A. Kosowsky, and A. Stebbins. A probe of primordial gravity waves and vorticity. Phys. Rev. Lett., 78:2058, 1997.

[16] U. Seljak and M. Zaldarriaga. Signature of gravity waves in the polarization of the microwave background. Phys. Rev. Lett., 78:2054, 1997.

[17] S. Chandrasekhar. Radiative Transfer. Clarendon Press, Oxford, 1950.

[18] W. Hu and M. White. CMB anisotropies: Total angular momentum method. Phys. Rev. D, 56:596, 1997.

[19] W. Hu, U. Seljak, M. White, and M. Zaldarriaga. A complete treatment of CMB anisotropies in a FRW universe. Phys. Rev. D, 57:3290, 1998. 
[20] A. Loeb and A. Kosowsky. Faraday rotation of microwave background polarization by a primordial magnetic field. Astrophys. J., 469:1, 1996.

[21] R. Durrer, T. Kahniashvili, and A. Yates. Microwave background anisotropies from Alfvén waves. Phys. Rev. D, 58:123004, 1998.

[22] G.F. Smoot et al. Structure in the COBE differential microwave radiometer first-year maps. Astrophys. J., 396:L1, 1992.

[23] S.W. Hawking. Perturbations of an expanding universe. Astrophys. J., 145:544, 1966.

[24] G.F.R. Ellis and M. Bruni. Covariant and gauge-invariant approach to cosmological density fluctuations. Phys. Rev. D, 40:1804, 1989.

[25] G.F.R. Ellis, J. Hwang, and M. Bruni. Covariant and gauge-independent perfect-fluid Robertson-Walker perturbations. Phys. Rev. D, 40:1819, 1989.

[26] A.D. Challinor. In preparation, 1999.

[27] J. Silk. Fluctuations in the primordial fireball. Nature, 215:1155, 1967.

[28] Z. Haimann and L. Knox. Reionization of the intergalactic medium and its effect on the CMB. Preprint astro-ph/9902311, 1999.

[29] J.M. Bardeen. Gauge-invariant cosmological perturbations. Phys. Rev. D, 22:1882, 1980.

[30] E.M. Lifshitz. Gravitational stability of the expanding universe. J. Phys. (Moscow), 10:116, 1946.

[31] R. Maartens and B.A. Bassett. Gravito-electromagnetism. Class. Quantum Grav., 15:705, 1998.

[32] M. Kamionkowski and A. Kosowsky. Detectability of inflationary gravitational waves with microwave background polarization. Phys. Rev. D, 57:685, 1998.

[33] M. Zaldarriaga, U. Seljak, and E. Bertschinger. Integral solution for the microwave background anisotropies in non-flat universes. Astrophys. J., 494:491, 1998.

[34] U. Seljak and M. Zaldarriaga. A line of sight integration approach to cosmic microwave background anisotropies. Astrophys. J., 469:437, 1996. 